\title{
Análisis del Entorno en la Gestión Universitaria: Una Aproximación desde la Teoría de Sistemas Sociales
}

Environment analysis in university management: an outline from social systems theory

\author{
Volumen 21, Número 1 \\ Enero - Abril \\ pp. 1-22
}

César Cisternas Irarrázabal 


\section{Análisis del Entorno en la Gestión Universitaria: Una Aproximación desde la Teoría de Sistemas Sociales \\ Environment analysis in university management: an outline from social systems theory}

\section{César Cisternas Irarrázabal ${ }^{1}$}

Resumen: Durante las últimas décadas, las universidades latinoamericanas han profundizado su integración al sistema universitario mundial, lo que ha implicado transformaciones radicales y tendencias a la complejización. El presente ensayo concibe la universidad como un sistema organizacional complejo, cuya reproducción tiene lugar mediante la toma de decisiones. Desde esta perspectiva se plantea que, ante un contexto de creciente complejidad e incertidumbre como el latinoamericano, la observación del entorno a través del análisis institucional comprende una herramienta fundamental para neutralizar potenciales procesos de desintegración que amenacen a la universidad. Se considera, en este sentido, que: (1) los modelos de gestión tradicional asumen un entorno estable en el futuro, (2) tales esquemas disminuyen la capacidad de la universidad de reaccionar a cambios externos, pudiendo, además, derivar en la rutinización del proceso de toma de decisiones, (3) ante la incertidumbre, la posibilidad de adaptarse/responder al entorno resulta fundamental para la sustentabilidad institucional, y (4) aquella capacidad solo existe si se incorpora el análisis del entorno al proceso de toma de decisiones. La argumentación se desarrolla tomando como caso de análisis el subsistema universitario chileno. Se concluye que para operar en el contexto actual, la universidad requiere transitar hacia modelos de gestión basada en evidencia, en cuanto entregan mayor capacidad para reducir la complejidad creciente del entorno general y competitivo de la universidad.

Palabras clave: gestión educacional, organización, toma de decisiones, sistema social, enseñanza superior.

Abstract: Throughout the last decades, Latin American universities have strengthen their integration into the global university system, which has implied radical transformations and trends toward complexification. This essay comprehends the university as a complex organizational system, which reproduces itself through decision-making. From this perspective, it is set out that an observation of the environment deploying institutional research is a fundamental tool to neutralize potential disintegration processes which could threaten university when faced with increasingly uncertain contexts as the Latin American one. In this sense, the following premises are taken into account: (1) traditional management models presume a stable environment in the future, (2) such schemes reduce the university's capacity to react to external changes, and besides, it may derive into the routinization of the decisionmaking process, (3) in uncertain contexts, the possibility of adapt/respond to the environment is essential for the institutional sustainability, and (4) that capacity only exist if the decision-making process involves an environment analysis. The argumentation is carried out using the Chilean university subsystem as analysis case. The conclusions suggest that to operate efficiently in the actual context, universities require to transit to evidence-based management models, since they offer a greater capacity to reduce the increasing complexity of the general and competitive university's environments.

Key words: educational management, organization, decision-making, social system, higher education.

\footnotetext{
${ }_{1}^{1}$ Docente de la Universidad de La Frontera, Temuco, Chile. Sociólogo y Magíster en Ciencias Sociales por la Universidad de La Frontera, Temuco, Chile. ORCID: https://orcid.org/0000-0002-4510-4239
}

Dirección electrónica: c.cisternas.irarrazabal@gmail.com

Ensayo recibido: 16 de junio, 2020

Enviado a corrección: 8 de setiembre, 2020

Aprobado: 19 de octubre, 2020 


\section{Introducción}

En los últimos veinte años, la demanda por educación superior en América Latina se ha incrementado sostenidamente. Uno de los principales factores que explican estas cifras es el aumento de las tasas de egreso de enseñanza media, que ha producido un flujo mayor de jóvenes en condiciones de postular a la educación terciaria (Avitabile, 2017). Chile no ha quedado exento a esta tendencia, en efecto, ha alcanzado el estadio de universalización de la educación superior, es decir, ostenta una tasa de matrícula bruta superior al 50\% (Brunner y Miranda, 2016; Brunner y Pedraja-Rejas, 2017).

Desde el año 2000, la matrícula en universidades ha crecido un $72 \%$, pasó, así, de 435.884 estudiantes en el 2000, a 749.149 en el 2019 (Servicio de Información de Educación Superior, 2010; 2019). La expansión de la oferta, derivada del aumento de programas e instituciones, ha sido clave en proveer al subsistema universitario chileno la capacidad para absorber la demanda.

Esta universalización de la formación superior, junto al establecimiento de criterios de calidad orientados a regular la oferta, los mecanismos de financiamiento por desempeño y otros factores, conforman un escenario marcado por una complejización creciente del entorno universitario y de las propias instituciones. Sin embargo, las profundas modificaciones del entorno no han generado, en las universidades, una reorientación de las formas tradicionales de gestión. Los lineamientos estratégicos de las instituciones continúan siendo definidos en el marco de procesos altamente autorreferenciales (Rodríguez-Ponce y Pedraja-Rejas, 2013), al tiempo que las capacidades de análisis del entorno externo e interno no se han desarrollado adecuadamente (DPM Research, 2016). Lo anterior constituye una sensible falencia de los modelos de gestión universitarios en Chile, en cuanto la capacidad de adaptarse a un entorno cambiante constituye la clave de la supervivencia, en el largo plazo, de cualquier organización (Zimmermann, 2011).

Desde una perspectiva sistémica, la universidad, en tanto organización, es concebida como un sistema social que opera mediante decisiones (Luhmann, 2006; 2010). Así, la toma de decisiones constituye la actividad primordial a partir de la cual esta institución se reproduce a sí misma, distinguiéndose de su entorno. Adoptando este enfoque, el presente ensayo reflexiona en torno a la creciente incertidumbre que enfrenta la universidad en su operación, y la relevancia que adquiere la mirada al entorno en la gestión de este tipo de organizaciones. Se plantea, así, desde una perspectiva sistémica, que ante un contexto de creciente complejidad e incertidumbre, la observación del entorno a través del análisis institucional 
comprende una herramienta fundamental para neutralizar potenciales procesos de desintegración que amenazan a la universidad.

Tras esta tesis se encuentra una serie de consideraciones o premisas respecto a la relación entre la universidad, como sistema organizacional, y su entorno, a saber: (1) los sistemas de gestión tradicional suponen un entorno estable en el mediano plazo, (2) la adhesión a tales modelos priva a la universidad de la capacidad de responder a las condiciones del entorno y, además, puede derivar en la rutinización del proceso de toma de decisiones, (3) la respuesta o adaptación al entorno resulta clave para la sustentabilidad universitaria en un contexto de alta incertidumbre, y (4) solo se puede generar esta capacidad de respuesta/adaptación mediante la incorporación del análisis del entorno al proceso de toma de decisiones.

Se ha considerado el subsistema universitario chileno como caso de análisis, con el fin de ilustrar, por una parte, el modo en que la perspectiva teórica adoptada puede aplicarse al plano empírico y, por otra parte, el grado de desarrollo del análisis institucional en el subcontinente.

El trabajo se divide en cuatro secciones. La primera de ellas se enfoca en problematizar la universidad como organización desde la teoría de sistemas sociales. Se pondrá un especial énfasis, en este apartado, en interpretación sistémica de la relación universidad-entorno y en la exposición de las premisas que subyacen a la tesis planteada en el ensayo. Por su parte, la segunda sección da cuenta de la realidad de la gestión universitaria en Chile y el lugar que el análisis del entorno tiene en esta. La tercera sección se orienta a exponer el modo en que el análisis institucional puede encarnar una estrategia para incorporar la consideración del entorno en el proceso de toma de decisiones. Finalmente, se presentan las conclusiones del trabajo.

\section{La universidad como un sistema organizacional complejo}

\subsection{La concepción de la universidad desde la teoría de sistemas sociales}

La sociedad moderna se distingue de otras formas sociales por su creciente diferenciación funcional. Este rasgo estructural posibilita a la sociedad moderna, como sistema, a lidiar con un alto grado de complejidad a través de subsistemas comunicacionales orientados a una función particular (Luhmann, 1998). En este contexto único, proporcionado por la modernidad, emerge la organización como forma social. 
La organización es un sistema social autopoiético que opera en función de la toma de decisiones. Es decir, la organización reproduce por sí misma la comunicación que la constituye a partir del encadenamiento de decisiones (Luhmann, 2010). Estos sistemas sociales le permiten a la sociedad procesar las grandes cantidades de incertidumbres derivadas de su complejización. Esa incertidumbre es procesada mediante la toma de decisiones, proceso en el cual ciertas opciones de elección son excluidas en la selección que implica la operación de decisión (Luhmann, 2006).

La universidad es unas de las organizaciones características de la sociedad moderna. Esta presenta un alto grado de complejidad, en cuanto responde a los requerimientos de tres sistemas sociales: el científico, pues constituye el principal espacio de generación y reproducción del conocimiento científico; el educacional, ya que se orienta a la trasmisión de conocimiento en la modalidad de educación terciaria; y el económico, debido a que entrega certificaciones de las aptitudes que requiere parte del mercado laboral (Chávez, 2011).

Actualmente, las instituciones universitarias de todo el mundo enfrentan nuevos desafíos, entre los cuales están los recortes presupuestarios, transformaciones de la estructura académica y de gobernanza, avance tecnológico acelerado, reforzamiento de los sistemas de aseguramiento de la calidad y el balance entre las misiones de enseñanza, investigación y servicio (Webber y Calderon, 2015). En un escenario en que la educación superior ha ido dejando atrás progresivamente los límites nacionales para constituirse en un sistema mundial (Pfeffer y Stichweh, 2015), las universidades latinoamericanas han comenzado a sufrir las mismas presiones a lo largo de las últimas dos décadas. Desde entonces, se acentuó la incorporación del subcontinente a las redes globales de procesos productivos, territorios e individuos propias de lo que Castells (2010) denomina sociedad de la información. En este marco, las transformaciones en el mercado del trabajo, las nuevas tendencias demográficas y el desplazamiento en los énfasis de producción de conocimiento (vinculación ciencia-empresa, big science) incrementan la complejidad de los sistemas a los cuales la universidad se acopla. En consecuencia, se ve obligada a repensar sus procesos de enseñanza, vínculos con el mundo laboral y estrategias de producción científica.

Emplear un enfoque sistémico para el análisis de la gestión universitaria permite visualizar que las modificaciones en el entorno constituyen estímulos para la institución, pero que estos deben ser observados y procesados por las operaciones internas para tener efectos dentro del sistema universidad (Pfeffer y Stichweh, 2015). En consecuencia, este acercamiento teórico explicita que, ante las rápidas transformaciones que afronta, el éxito de 
la gestión universitaria depende de su capacidad de adaptación (respuesta) al entorno, la cual tiene lugar siempre desde su autorregulación.

\subsection{Una perspectiva sistémica de la relación universidad-entorno}

Tal como lo plantea Morin (2005), la organización tiende naturalmente a degradarse, siendo el repensarse con regularidad la única manera de evitar este curso. En otras palabras, su pervivencia se juega "en la aptitud del conjunto de la organización de regenerarse y reorganizarse haciendo frente a todos los procesos de desintegración" (Morin, 2005, p. 126).

En esa misma línea, Luhmann (2006) señala que la dinámica autopoiética de un sistema organizacional, que opera en función de la toma de decisiones basadas en decisiones pasadas, desarrolla una inclinación a la rutinización del proceso. Esto implica que la organización, debido a sus propias lógicas de operación, es proclive al decidir no decidir. Esto es, a la mantención del status quo, pero optar por no reevaluar periódicamente la estrategia institucional a la luz de potenciales modificaciones en las condiciones de desempeño (decidir no decidir) pone a la universidad en peligro de sucumbir ante procesos de desintegración.

La configuración de un sistema universitario mundial, gatillada por la globalización, tiende a aumentar progresivamente la complejidad del entorno de la universidad. Como consecuencia, desde dicho entorno pueden emerger megatendencias, amenazas $u$ oportunidades claves para la sustentabilidad o estabilidad de la universidad. $Y$, dado que la reproducción del sistema universidad consiste, precisamente, en el trazar una distinción entre este y el entorno (Luhmann, 2006), la desatención a determinados fenómenos que tienen lugar en este último puede generar procesos de desintegración organizacional debido a la no respuesta/adaptación a un entorno de alta incertidumbre.

De esta manera, la modalidad que adquiere la operación autopoiética del sistema se convierte en un punto crítico. Los programas clásicos de gestión, como lo indica Morin (2005), se limitan a detallar una concatenación de acciones establecida a priori, suponiendo un entorno estable en el mediano plazo. En consecuencia, constituyen un derrotero rígido en cuanto a las decisiones de la organización con respecto a sus movimientos tácticos generales, limitando la posibilidad de responder a modificaciones del entorno que no se condigan con el escenario que sirvió como base para el diseño del programa.

El seguimiento de un curso preestablecido de decisiones inadecuado a las variaciones contextuales condenaría a la universidad a la zozobra o al desmejoramiento de sus condiciones de operación (pérdida de posicionamiento en la competencia, disminución de la 
matrícula, fuga de académicos, disminución de los recursos económicos disponibles, entre otros). No obstante, en la realidad es poco probable que, ante cambios profundos en el entorno, una institución persista en aplicar al pie de la letra las selecciones contenidas en su plan estratégico -o cualquier instrumento que defina un programa de acción-, pues sería una falencia profunda del sistema de gestión.

La tendencia en estos casos será que los cuerpos directivos -o quienes estén en condiciones de tomar decisiones de alcance estratégico en la organización- orienten la concatenación de decisiones en otra dirección -decidiendo, así, no decidir lo que previamente se había evaluado como decisiones correctas-. Así, se generaría una recursividad del proceso de decisión que revisaría las selecciones realizadas en instancias previas (Luhmann, 2006). Ahora bien, esta orientación del sistema presenta el riesgo de conducir a la organización por un rumbo igualmente incorrecto, como consecuencia de una distorsión en la lectura de las condiciones de operación de la institución. Aquí, la decisión de reducir complejidad a partir de un análisis reduccionista del entorno constituiría el principal factor distorsionador del proceso.

Ante contextos altamente variables, entonces, los modelos más eficientes de autorregulación organizacional serían aquellos que entreguen mayor flexibilidad para la toma de decisiones, pero que, al mismo tiempo, faciliten la anticipación a transformaciones del escenario en que opera la universidad. Esto se debe a que el acoplamiento estructural de la universidad a entornos de alta incertidumbre requiere que esta cuente con una profunda capacidad de respuesta o adaptación, de modo que su sustentabilidad institucional no se vea en riesgo. De manera que la configuración de estrategias emerge como un mejor esquema de gestión, por cuanto implica un ejercicio reflexivo y plástico de proyección de escenarios probables que pone a disposición de la organización herramientas para hacer frente a contextos inciertos (Morin, 2005).

Para poder identificar potenciales amenazas y operar en el marco de su multiplicidad de acoplamientos estructurales simultáneos, la universidad tenderá a incrementar su complejidad interna (instalar nuevas unidades, construir capacidades de análisis, crear más puestos de analista, entre otros). Esto se debe a que el único modo de que el sistema sea capaz de procesar una mayor complejidad del entorno es contar con estructuras que puedan reducir eficientemente tal complejidad (Luhmann, 1995; 2006). Bajo estas condiciones operacionales, que permiten la incorporación del análisis del entorno a la toma de decisiones, la universidad estará mejor preparada para responder o adaptarse a su entorno. 
La complejización de la universidad, entonces, no solo tiene que ver con las respuestas al sistema educativo, científico y económico. La relación de la universidad con el entorno no se limita a formar nuevos profesionales, generar conocimiento y certificar las aptitudes necesarias para el mercado del trabajo calificado, sino que también considera las condiciones de dicho entorno: la competencia, la regulación, el financiamiento, el prestigio, entre otros. En este sentido, la naturaleza del entorno universitario comprende un sinnúmero de variables, por lo cual su consideración en procesos de toma de decisiones requerirá una interpretación informada de dicho entorno, y puesto que el conocimiento y la evidencia constituyen estructuras que guían la observación (Knudsen, 2017), mediante el desarrollo de capacidades analíticas, la universidad logrará una orientación flexible hacia el entorno, lo cual le permitirá responder a sus contingencias.

\section{El entorno en la gestión universitaria en Chile}

América Latina ha sido una de las regiones que ha presenciado un crecimiento más vertiginoso de sus universidades en las dos últimas décadas. En este lapso se ha incrementado considerablemente la matrícula de pregrado, el número de profesionales que continúa estudios de postgrado, la oferta de programas de formación (Avitabile, 2017; Oficina Regional de Educación para América Latina y el Caribe, 2013), así como la producción científica ${ }^{2}$.

Con respecto a la gestión, las universidades latinoamericanas no han respondido de la mejor manera ante estos desafíos que han emergido en su entorno. La investigación de Rodríguez-Ponce y Pedraja-Rejas (2009) sobre su dirección estratégica, muestra que, en general, llevan a cabo procesos de gestión que no son rigurosos ni consiguen trazar e implementar estrategias que respondan a las condiciones del entorno y a las capacidades disponibles. Por su parte, Barreda (2016) evidencia que, en la mayoría de los casos, se emplean metodologías de planificación estratégica desactualizadas y se configuran objetivos estratégicos que no se relacionan completamente con el diagnostico organizacional efectuado.

En este marco, es cuestionable el potencial de la mayoría de las universidades latinoamericanas para poder responder satisfactoriamente a las condiciones del entorno. Las debilidades de sus modelos de gestión se transforman en un punto crítico, pues los riesgos

\footnotetext{
2 Según datos del sistema SciVal de Elsevier, la producción científica de la región (considerando artículos y revisiones Scopus) prácticamente se ha quintuplicado entre 2000 y 2019.
} 
del entorno pueden llegar a determinar el fracaso de los lineamientos estratégicos de una organización (Galarza y Almuiñas, 2015).

El panorama en Chile no dista mucho de la realidad general de América Latina. Las instituciones del país son capaces de identificar algunas de las principales amenazas del entorno y sus propias ventajas comparativas; sin embargo, no logran consolidar estrategias sostenibles (Rodríguez-Ponce y Pedraja-Rejas, 2013). Esta es una de las dimensiones en que se manifiestan las deficiencias en cuanto a la coherencia del diagnóstico del entorno realizado y la estrategia organizacional (Rodríguez-Ponce y Pedraja-Rejas, 2013).

En el año 2016, una consultoría externa sobre los sistemas de gestión de las universidades chilenas mostró un escaso desarrollo en cuanto a las capacidades de análisis del entorno externo e interno, que, en general, son de carácter descriptivo y reactivo (DPM Research, 2016). Si bien en los años recientes se han producido avances en cuanto a la gestión del conocimiento asociado a los procesos de toma de decisiones (Araya-Guzmán, Henríquez, Ramírez-Correa y Barra, 2019), estos aún tienen un alcance restringido dentro de las universidades.

El limitado análisis respecto a la complejidad del entorno y sus contingencias resulta amenazante para las universidades chilenas, en un país en que los procesos de complejización transversales a la realidad latinoamericana se han dado con particular fuerza. En este sentido, el entorno de la universidad chilena destaca en el contexto regional por una acentuada expansión de la oferta de programas de pre y postgrado, el crecimiento de la producción científica y el aumento de la participación en la educación superior. La confluencia de estos factores ha provocado una fuerte competencia en el área y la configuración de un contexto en que los programas rígidos de acción a largo plazo resultan insostenibles debido a la alta contingencia del entorno.

Lo anterior se suma a una serie de rápidos cambios que han tenido lugar en múltiples ámbitos a lo largo de los últimos años. Entre las principales transformaciones se pueden mencionar: (a) ajustes de los marcos regulatorios, (b) consolidación del sistema de evaluación de la calidad, al tiempo que más instituciones obtienen mayores niveles de acreditación, (c) mayor cantidad de universidades que afianzan su acoplamiento estructural con el sistema ciencia a través de la orientación hacia la producción de investigación ${ }^{3}$, y (d) introducción de

\footnotetext{
3 Actualmente 31 universidades cuentan con una producción superior a 100 artículos anuales indexados en Web of Science en 2019 (Consejo Nacional de Educación, 2020), mientras que 25 se encuentran acreditadas en investigación, según información disponible en la página web de la Comisión Nacional de Acreditación al 10 de julio de 2020, la que puede ser consultada en: https://www.cnachile.cl/Paginas/buscador-avanzado.aspx
} 
la internacionalización como nuevo desafío para la universidades del país (Geldres, Vásquez y Ramos, 2013). A esto se agregan, en el caso de las instituciones estatales, los desafíos asociados al financiamiento sujeto a desempeño (Muñoz, 2013; Sisto, 2017) y a un estricto marco regulatorio legal que vuelve aún más crítico el diseño de una estrategia organizacional orientada al logro de los objetivos propuestos (Villegas y Valderrama, 2018).

Todos estos factores impactan la universidad, limitando sus posibilidades de elección y llevándola a replantearse las decisiones tomadas. Su capacidad de respuesta al entorno es clave en la resistencia exitosa ante la tendencia propia de todo sistema organizacional a ser degradado por el entorno en caso de inacción o de decidir no decidir respecto a este (Morin, 2005; Zimmermann, 2011). No parece conveniente, entonces, para las universidades chilenas, mantener los modelos de gestión autorreferenciales hasta ahora implementados, que las sitúan en una posición preeminentemente pasiva ante el entorno.

\section{El análisis institucional y la observación del entorno universitario}

\subsection{Análisis institucional como estrategia para la gestión basada en evidencia}

Ante contextos como el chileno, de alta incertidumbre generada por una creciente complejidad en el entorno, particularmente en los sistemas educacional, jurídico, científico y político, resulta fundamental el sondeo de dicho entorno y la anticipación a sus cambios, a través de lineamientos organizacionales que aumenten la capacidad de adaptación de la universidad.

Hace algunas décadas, distintas aproximaciones a la gestión organizacional han tendido a confluir en la misma idea que se desprende del análisis sociológico sistémico de la relación organización-entorno: la organización debería considerar el entorno en el proceso de toma de decisiones, base de su reproducción autopoiética (véase Chermack, 2011; Martelli, 2014; Zimmermann, 2011).

Una de las perspectivas que ha incorporado la observación de la relación organizaciónentorno es el modelo de gestión basado en evidencia, el cual surge desde el intento de trasladar la práctica de la medicina basada en evidencia al ámbito de la gestión organizacional (Morell, 2008; Reay, Berta y Kohn, 2009; Scheibe, Nilakanta, Ragsdade y Younie, 2019). Esta aproximación a la gestión sugiere aplicar los resultados de la investigación empírica a la resolución de problemas que enfrente una organización (Rousseau, 2006). Otros autores, ampliando la definición, la han caracterizado como la fundamentación del curso de acción institucional en la mejor evidencia disponible (Pfeffer y Sutton, 2006) o como la consideración 
en la toma de decisiones y definiciones estratégicas de la evidencia de mayor calidad a la que se pueda acceder (Briner, Denyer y Rousseau, 2009). Por su parte, Transfield, Denyer y Smart (2003) emplean el concepto de decisiones informadas por evidencia, asumiendo que la evidencia es, a veces, incompleta e insuficiente para seleccionar algún curso de acción.

El conocimiento consiste en estructuras que guían la observación (Knudsen, 2017). Por ende, la gestión basada en evidencia implica la introducción de estructuras -constituidas desde observaciones previas del entorno- que impacten las observaciones realizadas en el marco de la operación de la organización. En este sentido, la adopción de fórmulas de gestión basada en evidencia permitiría disminuir el riesgo de rutinización de las decisiones en la autorreferencia organizacional a través del incentivo a la observación del entorno. Además, tal como lo plantea Rousseau (2006), su puesta en práctica, al recolectar información sobre el desempeño institucional y el efecto de las decisiones tomadas, permite el aprendizaje institucional y la mejora continua, contribuyendo también a que las estrategias adoptadas por los tomadores de decisiones incrementen su legitimidad dentro de la organización.

En el ámbito universitario, los principios de la gestión basada en evidencia se han incorporado, hace varias décadas, mediante el análisis institucional (institutional research). Esta práctica consiste en la realización de estudios acotados dentro de una universidad con el fin de proveer información que sustente la definición de estrategias institucionales, la formulación de políticas y la toma de decisiones en general (Saupe, 1990). En una delimitación más reciente, Webber y Calderón (2015) han descrito la investigación institucional como un conjunto de funciones, actividades y roles ejecutados con el fin de ayudar a tomar decisiones basadas en evidencia.

Esta alternativa para la reducción de complejidad del entorno demanda la complejización interna de la universidad. En efecto, el análisis institucional es responsabilidad de unidades especializadas dentro de la organización -cuya conformación requiere nuevo personal, competencias, herramientas de análisis, recursos, entre otros-.

En América Latina este tipo de unidades emergen en los noventa, ligadas principalmente a la evaluación y el aseguramiento de la calidad (Saavedra, Pita-Carranza y Opazo, 2015). En Chile, el Ministerio de Educación implementó, desde el año 2006, fondos concursables para la planificación estratégica y el aseguramiento de la calidad, contexto en que muchas universidades crearon o fortalecieron sus unidades de análisis (Reich et al., 2011). Sin embargo, estas oficinas no han adquirido la legitimidad suficiente entre las restantes unidades 
de los planteles, al tiempo que no han logrado integrar los distintos sistemas de datos institucionales (Saavedra et al., 2015).

Estos factores limitan drásticamente la efectividad de un enfoque de gestión basado en evidencia, en cuanto se ha demostrado que el impacto de la analítica de datos en la calidad de las decisiones tomadas en una organización se correlaciona directamente con el grado de intercambio de conocimiento al interior de esta (Ghasemagheai, 2019). Además, dichos factores impiden el aprovechamiento en beneficio de la organización de aquello que se ha descrito como dark data, es decir, aquellos datos que se recolectan en el marco de los procesos propios, pero que no son analizados para mejorar las decisiones (Ajis y Baharin, 2019; Hand, 2020). De este modo, las unidades de análisis chilenas se enfrentan al desafío al igual que en la mayor parte del subcontinente- de carecer de una cultura en que la toma de decisiones se base en evidencia y análisis (Saavedra et al., 2015).

Transitar hacia esquemas de gestión que ponen el acento en la evidencia presenta grandes ventajas para una organización como la universidad. Ayuda, en gran medida, a contrarrestar la incertidumbre, el conflicto y la ambigüedad asociados a la toma de grandes decisiones (Madhavan y Mahoney, 2012). A pesar de esto, existen al menos dos características inherentes a las dinámicas de la universidad -y de toda organización- y su relación con el entorno, que limitan la aplicabilidad de esta forma de gestión. La primera se vincula a la incapacidad de obtener evidencia sobre algunas temáticas, mientras que la segunda corresponde a la premura con que deben ser tomadas ciertas decisiones en algunos casos.

En tal marco, se debe dejar claro que este enfoque de gestión no designa como evidencia solo a aquella producida mediante investigación, estudios o análisis, sino que considera también la experiencia que los tomadores de decisiones han adquirido en el ejercicio de sus labores. A su vez, reconoce la existencia de limitaciones en cuanto a la evidencia que puede estar disponible para la toma de decisiones (Briner et al., 2009; Martelli y Hayirli, 2018; Transfield et al., 2003). Así, según Barends, Rousseau y Briner (2014), la práctica de la gestión basada en evidencia exige el escrutinio de cuatro fuentes de evidencia: científica, organizacional (datos internos), experiencial y la percepción de los grupos de interés.

De acuerdo con Martelli y Hayirili (2018), debido a que los sistemas sociales son abiertos y dinámicos, y a que la sociedad tiende hacia la complejidad y la incertidumbre, lo que define la calidad de la evidencia no es su ajuste a cierto ranking o a criterios preestablecidos, sino la variedad de las fuentes desde las que se obtiene. En este sentido, los profesionales de análisis 
y los tomadores de decisiones deben ser conscientes de que, en ciertas ocasiones, la mejor alternativa será recurrir a la evidencia disponible en el momento, absteniéndose de una auscultación exhaustiva del entorno (Knudsen, 2017). El camino contrario llevaría solo al aumento de la complejidad interna y a una incapacidad de reducir la complejidad del entorno. En consecuencia, la capacidad estratégica de la universidad, inmovilizada, se vería imposibilitada de abordar la contingencia.

Consolidar el tránsito hacia este tipo de enfoques de gestión es una tarea ardua, para lo cual resulta clave la instalación de capacidades (Webber, 2018), además de la configuración de una cultura de evidencia (Goomas, 2014) o cultura de toma decisiones guiadas por la investigación (Chirikov, 2013). Esta última implica la difusión del convencimiento de que la recolección de evidencia disponible y el análisis del entorno -externo, pero también internoconduce a la toma de mejores decisiones. Esta cultura puede llegar a ser difícil de conformar, tal como en el caso chileno (Saavedra et al., 2015), pero algunos factores facilitadores, en este sentido, pueden ser la disponibilidad de los resultados de los análisis para los distintos miembros de la universidad, la existencia de repositorios de datos centralizados y el acceso a la información mediante interfaces amigables al usuario (Goomas, 2014).

\subsection{Los niveles del análisis del entorno universitario}

Como se mencionó anteriormente, uno de los pilares de los enfoques de gestión que consideran fundamental la evidencia para la toma de decisiones es el análisis del entorno. En el caso particular del análisis institucional, uno de los lineamientos clave es tener siempre en consideración la misión de la institución, sus objetivos y sus diversas relaciones con el contexto social, económico, geográfico en el que opera (Webber y Calderón, 2015; Webber, 2018).

Dada la incertidumbre creciente del entorno de las universidades -tanto a nivel internacional, pero sobre todo a nivel nacional-, una técnica de particular utilidad es la proyección de escenarios. Aquella busca observar y procesar la complejidad del entorno a través de la elaboración de un conjunto de contextos contingentes posibles, intentando así, anticiparse a los escenarios que, de acuerdo con la evidencia disponible y a reflexiones proyectivas, pudiesen resultar los más probables (Chermack, 2011). Los escenarios resultantes de este ejercicio no son predicciones cuantitativas. Pueden estar basados en ellas, pero tienen un carácter descriptivo e incluyen información de otras fuentes diferentes a las proyecciones estadísticas (Martelli, 2014). 
Para la perspectiva sistémica, la organización es fundamentalmente una distinción entre el entorno y las operaciones de decisión que dan lugar a las estructuras comunicacionales que la constituyen como sistema (Luhmann, 2006; 2010). Desde este punto de vista, el entorno organizacional comprende un conjunto muy amplio de sistemas y estructuras comunicacionales. Por este motivo, para facilitar su análisis y la proyección de escenarios futuros suele dividírselo en dimensiones o niveles. Una de las distinciones más empleadas a este respecto se vincula con el ámbito geográfico, que a menudo conduce a la división entre el entorno nacional (o regional) y el global. Sin embargo, tal como lo sugieren Pfeffer y Stichweh (2015), dado el carácter globalizado de la sociedad y la interrelación cada vez mayor entre las universidades de distintas partes del mundo, la idea de sistemas de educación superior nacionales se vuelve insostenible a nivel empírico. Aquella división geográfica puede emplearse como diferenciación analítica en el estudio de ciertos problemas, pero teniendo en cuenta sus limitaciones y las potenciales distorsiones que puede inducir.

Otra alternativa de mayor utilidad para diferenciar niveles analíticos en el entorno es trazar una distinción con base en ciertas características estructurales. A este respecto, Martelli (2014) distingue entre el microentorno o entorno de operación, que incluye todos aquellos elementos que son más cercanos a la organización y sus actividades, y el macroentorno, que comprende todas aquellas estructuras generales y condiciones que impactan en la operación de la organización. Empleando una conceptualización diferente, Chermack (2011) sugiere una segmentación que concibe, por una parte, un entorno contextual, caracterizado por la presencia de las fuerzas sociales, tecnológicas, económicas, medioambientales y políticas; y por otra, un sistema de desempeño, en el cual se encuentran los competidores de la organización y sobre el cual se despliegan sus fuerzas competitivas.

Ambas perspectivas trazan distinciones que confluyen en un punto: el diferenciar analíticamente el entorno social general y aquel en que se encuentran otras organizaciones que ofrecen las mismas prestaciones o que persiguen un objetivo similar. Cada uno de estos entornos le ofrece, básicamente, dos posibilidades a una organización: establecer o suprimir determinados acoplamientos estructurales -esto es, entrar o salir de ciertos entornos específicos- o bien acotar/incrementar las posibilidades de selección del sistema en su operación autopoiética -esto es, el rango de opciones entre las que se puede optar- (Child, 1997).

No obstante, ambos entornos presentan ciertas particularidades para el análisis institucional. Se debe tener en cuenta, en esta línea, que el entorno general de la universidad 
cambia constantemente, elevando su complejidad y, de este modo, sus niveles de incertidumbre. Por tanto, el análisis de este resulta una prioridad para las instituciones en cuanto a la proyección de escenarios (Chermack, 2011). Y es que, como lo señala Torres (2016, p. 663):

[...] para describir la complejidad y la contingencia universitaria, es necesario previamente, ubicarla en la relación universidad/sociedad, adoptando marcos de análisis que consideren no sólo las operaciones internas que efectúan las organizaciones educativas superiores, sino también las dinámicas sociales e históricas.

La atención a este entorno adquiere particular relevancia en un contexto como el latinoamericano, en el cual la diferenciación sistémica no es policéntrica, sino concéntrica (Mascareño, 2003). Esta lógica impacta en que se generen jerarquías entre los sistemas, derivando en una pérdida de autonomía de los sistemas subordinados, los que tienden a incluir en sus operaciones códigos de otros sistemas -muy a menudo aquellos del sistema económico o político- (Mascareño, 2003).

Por su parte, el examen del sistema o entorno competitivo demanda la evaluación del desempeño de la organización. En este propósito, resulta necesaria la observación comparada de otras instituciones o de unidades internas, mediante un ejercicio de benchmarking, el cual permite visualizar las dinámicas de competencia con el fin de establecer las posibilidades y restricciones de selección que se presentan en el entorno a este respecto.

En el ámbito de la educación superior, el benchmarking constituye una herramienta metodológica que puede ser de utilidad en la identificación de buenas prácticas, el mejoramiento continuo y la identificación, adopción y logro de estándares de calidad (Tasopoulou y Tsiotras, 2017). En lo que respecta a la gestión universitaria, existe una amplia difusión de este acercamiento analítico (Alghamdi, Haider, Hewage y Sadiq, 2019; Cardozo, Barros, Quelhas, Filho y Salles, 2020; Harris et al., 2019; Salas-Velasco, 2019; Streiner y Besterfield-Sacre, 2019), que puede ser aplicado a aspectos internos de la institución (comparación de programas de formación, productividad de unidades académicas, entre otros) o enfocarse en la comparación del rendimiento institucional con el de otras universidades.

El benchmarking, vinculado al entorno externo, resulta fundamental para tener una perspectiva clara respecto al ámbito competitivo de la universidad. Se vuelve una herramienta de suma utilidad en cuanto al diseño de estrategias dirigidas a aumentar la calidad y la eficiencia de la institución (Levy y Ronco, 2012), en tanto entrega la posibilidad de, por ejemplo, 
focalizar la inversión en los ámbitos prioritarios en que la organización obtenga rendimientos más débiles o en aquellos en que cuente con ventajas competitivas. Al mismo tiempo, la visibilización de la posición de la universidad respecto a su competencia trasforma al benchmarking en una buena instancia para ayudar a derribar la resistencia al cambio dentro de esta, además de promover un compromiso mayor con la autoevaluación y la calidad institucional (Alstete, 1995).

Ambos niveles de análisis son necesarios para la construcción de una representación correcta del entorno. Únicamente un ejercicio analítico de tal naturaleza pondrá a disposición del sistema organizacional universitario el conocimiento o estructuras comunicativas que sustenten una recursividad de la operación de decisión adecuada para responder/adaptarse a las contingencias e incertidumbres del entorno organizacional.

\section{Conclusiones}

En las últimas décadas, el avance de la globalización ha consolidado una red global de procesos productivos, territorios e individuos, marco en el cual las fronteras nacionales de los sistemas de educación superior se han ido difuminando para constituir un sistema global. En este escenario, las universidades de todo el mundo enfrentan presiones similares desde sus entornos, pero muchas de ellas se presentan con más fuerza en América Latina debido a que su subsistema universitario ha sufrido una aceleración abrupta de su proceso de integración global desde comienzos del siglo XXI.

En el contexto chileno, a tales desafíos mundiales de la educación superior, se añaden factores -como la expansión del acceso, la proliferación de nuevos programas o la emergencia de universidades más complejas-, que incrementan la complejidad e incertidumbre del entorno.

Debido a la creciente complejidad de su entorno, la gestión de la universidad como sistema organizacional que se reproduce a sí mismo mediante la toma de decisiones se ha visto dificultada. Los esquemas tradicionales de gestión, predominantes en las instituciones de educación superior chilenas, incrementan el riesgo de rutinización del proceso de decisión y disminuyen la capacidad de adaptación o respuesta a las contingencias del entorno. De este modo, crece el riesgo de que las megatendencias, oportunidades y amenazas que emergen en el entorno se transformen en procesos de desintegración que amenacen la sustentabilidad de las universidades. 
En este sentido, los modelos de gestión basados en evidencia pueden generar estructuras para una observación del entorno que, de este modo, permitan acotar las posibilidades de selección relevantes para la universidad. Al mismo tiempo, tales estructuras pueden convertirse en una base para el diseño de marcos estratégicos flexibles que entreguen a la institución una mayor capacidad de responder o adaptarse adecuadamente a los constantes cambios de su entorno.

A nivel mundial, una de las estrategias seguidas por muchas universidades para implementar sistemas de gestión que consideran evidencia en la toma de decisiones es el potenciamiento del análisis institucional. Esta práctica, que consiste en la realización de estudios acotados relativos a una problemática de interés estratégico para la universidad, fomenta la observación heterorreferencial en la operación de toma de decisiones. En consecuencia, permite responder de mejor modo ante la incertidumbre derivada de la complejidad del entorno y los múltiples acoplamientos estructurales de la universidad.

Ahora bien, este modelo de gestión tiene considerables ventajas en cuanto a la configuración de estrategias de mediano y largo plazo, pero se ve limitado al momento de tomar decisiones impostergables y urgentes. En estos casos, se vuelve necesario abstenerse de realizar observaciones profundas del entorno, ante lo cual cobra relevancia otro tipo de evidencia: aquella experiencia acumulada por los tomadores de decisiones. En tales escenarios, esta es la única alternativa para no aumentar innecesariamente la complejidad interna de la universidad y evitar que su capacidad de operación se vea entorpecida.

\section{Referencias}

Ajis, Ahmad. y Baharin, Siti. (Abril, 2019). Dark data management as frontier of information governance. 9th Symposium on Computer Applications \& Industrial Electronics (ISCAIE), IEEE, Malasia. doi: https://doi.org/10.1109/ISCAIE.2019.8743915

Alghamdi, Abdulaziz., Haider, Husnain., Hewage, Kasun. y Sadiq, Rehan. (2019). Interuniversity sustainability benchmarking for Canadian higher education institutions: water, energy, and carbon flows for technical-level decision-making. Sustainability, 11(9), 1-26. doi: https://doi.org/10.3390/su11092599

Alstete, Jeffrey. (1995). Benchmarking in Higher Education: Adapting Best Practices to Improve Quality. Recuperado de https://www.ericdigests.org/1997-3/bench.html

Araya-Guzmán, Sergio., Henríquez, Carolina., Ramírez-Correa, Patricio. y Barra, Ana. (2019). Explorando la relación entre gestión del conocimiento y el rendimiento organizativo en instituciones de educación superior chilenas. Revista Ibérica de Sistemas e Tecnologias de Informação, (E17), 947-959. 
Avitabile, Ciro. (2017). The rapid expansion of higher education in the new century. En María Ferreyra, Ciro Avitabile, Javier Botero, Francisco Haimovich y Sergio Urzúa (Eds.), At a crossroads. higher education in Latin America and the Caribbean (pp. 47-75). Washington D.C.: Banco Mundial. Recuperado de http://hdl.handle.net/10986/26489

Barends, Eric., Rousseau, Denise., y Briner, Rob. (2014). Evidence-based management: the basic principles. Amsterdam: Center for Evidence-Based Management.

Barreda, Horacio. (2016). Planteamiento estratégico en universidades de América Latina. Revista Gestao Universitária na América Latina, 9(1), 257-277. doi: http://dx.doi.org/10.5007/1983-4535.2016v9n1p257

Briner, Rob., Denyer, Denise. y Rousseau, Denise. (2009). Evidence-based management: concept cleanup time? Academy of Management Perspectives, 23(4), 19-32. doi: https://doi.org/10.5465/amp.23.4.19

Brunner, José. y Miranda, Daniel. (2016). Educación superior en Iberoamérica. Informe 2016. Santiago: CINDA. Recuperado de https://cinda.cl/publicacion/educacion-superior-eniberoamerica-informe-2016/

Brunner, José. y Pedraja-Rejas, Liliana. (2017). Los desafíos de la educación superior en Chile. Ingeniare, 25(1), 2-7. doi: http://dx.doi.org/10.4067/S0718-33052017000100002

Cardozo, Nathália., Barros, Sergio., Quelhas, Osvaldo., Filho, Euricerio. y Salles, Wagner. (2020). Benchmarks analysis of the higher education institutions participants of the GreenMetric World University Ranking. En Walter Leal, Ubiratá Tortato y Fernanda Frankenberger (Eds.), Universities and sustainable communities: meeting the goals of the Agenda 2030 (pp. 667-683). Nueva York: Springer.

Castells, Manuel. (2010). The information age. Economy, society, and culture (Vol. I). The rise of the network society (2a ed.). Oxford: Wiley-Blackwell.

Chávez, Juan. (2011). Stitchkin o el educador. En Francisco Mujica y Francisco Torres (Comps.). Discursos iniciáticos: cátedras magistrales del rector David Stitchkin (pp. 5977). Santiago: Salieri.

Chermack, Thomas. (2011). Scenario planning in organizations. How to create, use, and assess scenarios. San Francisco: Berrett-Koehler Publishers.

Child, John. (1997). Strategic choice in the analysis of action, structure, organization and environment: retrospect and prospect. Organization Studies, 18(1), 43-76. doi: https://doi.org/10.1177/017084069701800104

Chirkov, Igor. (2013). Research universities as knowledge networks: the role of institutional research. Studies in Higher Education, 38(3), 456-469. doi: https://doi.org/10.1080/03075079.2013.773778

Consejo Nacional de Educación. (2020). Indicadores de investigación, años 2007-2016. Recuperado de https://www.cned.cl/indices/investigacion-anos-2007-2019 
DPM Research. (2016). Evaluación de los niveles de desarrollo de la gestión de la información en el sistema de educación chileno, medido a través de los niveles de desarrollo de las oficinas de análisis institucional (MECESUP 3-1484-SCC-CF). Recuperado de http://dfi.mineduc.cl/usuarios/MECESUP/File/2017/eventos/estudio-UUAAAIES/InformeFinal DPMR.pdf

Galarza, Judith. y Almuiñas, José. (2015). La gestión de los riesgos de planificación estratégica en las instituciones de educación superior. Revista Cubana de Educación Superior, 34(2), 45-53. Recuperado de http://scielo.sld.cu/scielo.php?script=sci arttext\&pid=S0257-43142015000200005

Geldres, Valeska., Vásquez, Fabiola. y Ramos, Heidy. (2013). Internacionalización de la educación superior en Chile: movilidad internacional de estudiantes en la Universidad de La Frontera. Revista Iberoamericana de Ciencia, Tecnología y Sociedad, 8(24), 45-62. Recuperado de http://www.revistacts.net/files/Volumen\%208\%20\%20N\%C3\%BAmero\%2024/FINAL/GeldresCORREG.pdf

Ghasemaghaei, Maryam. (2019). Does data analytics use improve firm decision making quality? The role of knowledge sharing and data analytics competency. Decision Support Systems, 120, 14-24. doi: https://doi.org/10.1016/j.dss.2019.03.004

Goomas, David. y Isbell, Teresa. (2014). The challenges of institutional research in building a culture of evidence: a case study. Community College Journal of Research and Practice, 39(5), 489-494. doi: https://doi.org/10.1080/10668926.2013.830581

Hand, David. (2020). Dark data: why what you don't know matters. Princeton: Princeton University Press.

Harris, Edwin., Langan, Mark., Barrett, Neil., Jack, Kirsten., Wibberley, Christopher. y Hamshire, Claire. (2019). A case for using both direct and indirect benchmarking to compare university performance metrics. Studies in Higher Education, 44(12), 22812292. doi: https://doi.org/10.1080/03075079.2018.1490893

Knudsen, Morten. (2017). From evidence-based management to management of nonknowledge. Emergence: Complexity and Organization, 19(2), 1-8. Recuperado de https://journal.emergentpublications.com/article/from-evidence-based-management-tomanagement-of-non-knowledge/

Levy, Gary. y Ronco, Sharron. (2012). How benchmarking and higher education came together. New Directions for Institutional Research, (156), 5-14. doi: https://doi.org/10.1002/ir.20026

Luhmann, Niklas. (1995). Introducción a la teoría de sistemas. México D.F.: Universidad lberoamericana.

Luhmann, Niklas. (1998). Complejidad y modernidad. De la unidad a la diferencia. Madrid: Trotta. 
Luhmann, Niklas. (2006). La sociedad de la sociedad. México D.F.: Herder/Universidad Iberoamericana.

Luhmann, Niklas. (2010). Organización y decisión. México D. F.: Herder/Universidad Iberoamericana.

Madhavan, Ravi. y Mahoney, Joseph. (2012). Evidence-based management in "macro" areas: the case of strategic management. En Denise Rousseau (Ed.), The Oxford handbook of evidence-based management (pp. 79-91). Oxford: Oxford University Press.

Martelli, Antonio. (2014). Models of scenario building and planning. Facing uncertainty and complexity. Hampshire: Palgrave Macmillan.

Martelli, Peter. y Hayirli, Tuna. (2018). Three perspectives on evidence-based management: rank, fit, variety. Management Decision, 56(10), 2085-2100. doi: https://doi.org/10.1108/MD-09-2017-0920

Mascareño, Aldo. (2003). Teoría de sistemas de América Latina. Conceptos fundamentales para la descripción de una diferenciación funcional concéntrica. Persona y Sociedad, 17(2), 1-20.

Morell, Kevin. (2008). The narrative of 'evidence based' management: a polemic. Journal of Management Studies, 45(3), 613-635. doi: https://doi.org/10.1111/j.1467$\underline{6486.2007 .00755 . x}$

Morin, Edgar. (2005). Introducción al pensamiento complejo. Barcelona: Gedisa.

Muñoz, Luis. (2013). Políticas públicas de financiamiento de la educación superior: implicancias para la universidad pública. Actualidades Investigativas en Educación, 13(3), 1-43. Recuperado de https://www.scielo.sa.cr/pdf/aie/v13n3/a21v13n3.pdf

Oficina Regional de Educación para América Latina y el Caribe. (2013). Situación educativa de América Latina y el Caribe: hacia la educación de calidad para todos al 2015. Santiago: ORELAC. Recuperado de http://www.unesco.org/new/fileadmin/MULTIMEDIA/FIELD/Santiago/images/SITIEDespanol.pdf

Pfeffer, Thomas. y Stichweh, Rudolf. (2015). Systems theoretical perspectives on higher education policy and governance. En Jeroen Huisman, Harry de Boer, David Dill y Manuel Souto-Otero (Eds.), The Palgrave international handbook of higher education policy and governance (pp. 152-175). Nueva York: Palgrave Macmillan.

Pfeffer, Jeffrey. y Sutton, Robert. (2006). Evidence-based management. Harvard Business Review, 84(1), 62-74. Recuperado de https://hbr.org/2006/01/evidence-based$\underline{\text { management }}$

Reay, Trish., Berta, Whitney. y Kohn, Melanie. (2009). What's the evidence on evidence-based management? Academy of Management Perspectives, 23(4), 5-18. doi: https://doi.org/10.5465/amp.23.4.5 
Reich, Ricardo., Machuca, Flora., López, Daniel., Prieto, Juan., Music, Juan., RodríguezPonce, Emilio. y Yutronic, Jorge. (2011). Bases y desafíos de la aplicación de convenios de desempeño en la educación superior de Chile. Ingeniare. Revista Chilena de Ingeniería, 19(1), 08-18. Recuperado de https://scielo.conicyt.cl/scielo.php?script=sci arttext\&pid=S0718-33052011000100002

Rodríguez-Ponce, Emilio. y Pedraja-Rejas, Liliana. (2009). Dirección estratégica en universidades: un estudio empírico en instituciones de lberoamérica. Interciencia, 34(6), 413-418. Recuperado de http://ve.scielo.org/scielo.php?script=sci arttext\&pid=S0378$18442009000600009 \& \operatorname{lng}=\mathrm{es} \& \mathrm{nrm}=\mathrm{iso} \& \operatorname{lng}=\mathrm{es}$

Rodríguez-Ponce, Emilio. y Pedraja-Rejas, Liliana. (2013). Dirección estratégica y calidad de las universidades: un estudio exploratorio desde Chile. Interciencia, 38(1), 35-41. Recuperado de https://www.redalyc.org/articulo.oa?id=33926506006

Rousseau, Denise. (2006). Is there such a thing as "evidence-based management"? Academy of Management Review, 31(2), 256-269. Recuperado de https://doi.org/10.5465/amr.2006.20208679

Saavedra, Mauricio., Pita-Carranza, María. y Opazo, Pablo. (2015). Institutional research in Latin America. En Karen Webber y Angel Calderon (Eds.), Institutional research and planning in higher education. Global contexts and themes (pp. 128-138). Londres: Routledge.

Salas-Velasco, Manuel. (2019). The technical efficiency performance of the higher education systems based on data envelopment analysis with an illustration for the Spanish case. Educational Research for Polivy and Practice, 19, 159-180. doi: https://doi.org/10.1007/s10671-019-09254-5

Saupe, Joe. (1990). The functions of institutional research. Tallahassee: Association for Institutional Research. Recuperado de https://files.eric.ed.gov/fulltext/ED319327.pdf

Scheibe, Kevin., Nilakanta, Sree., Ragsdale, Cliff. y Younie, Bob. (2019). An evidence-based management framework for business analytics. Journal of Business Analytics, 2(1), 4762. doi: https://doi.org/10.1080/2573234X.2019.1609341

Servicio de Información de Educación Superior. (2010). Evolución matrícula educación superior de Chile. Periodo 1990 - 2009. Recuperado de https://www.mifuturo.cl/wpcontent/uploads/2018/SIES/publicaciones/estudios/estudio evolucion matricula histori ca 1990 2009.pdf

Servicio de Información de Educación Superior. (2019). Informe matrícula 2017 en educación superior en Chile. Recuperado de https://www.mifuturo.cl/wpcontent/uploads/2019/07/Informe-Matricula-2019 SIES-1.pdf

Sisto, Vicente. (2017). Gobernados por los números: el financiamiento como forma de gobierno de la universidad en Chile. Psicoperspectivas, 16(3), 64-75. doi: http://dx.doi.org/10.5027/psicoperspectivas-vol16-issue3-fulltext-1086 
Streiner, Scott. y Besterfield-Sacre, Mary. (2019). Measuring the relative impact of international experiences on engineering students: A benchmarking analysis. International Journal of Engieneering Education, 35(5), 1503-1517.

Tasopoulou, Konstantina. y Tsiotras, George. (2017). Benchmarking towards excellence in higher education. Benchmarking, 24(3), 617-634. doi: https://doi.org/10.1108/BIJ-03$\underline{2016-0036}$

Torres, Emilio. (2016). Gobierno universitario en entornos de debate social en Chile. Observaciones desde la teoría de sistemas. Polis, 15(43), 655-680. doi: http://dx.doi.org/10.4067/S0718-65682016000100030

Transfield, David., Denyer, David. y Smart, Palminder. (2003). Towards a methodology for developing evidence-informed management knowledge by means of systematic reviews. British Journal of Management, 14(3), 207-222. doi: https://doi.org/10.1111/1467$\underline{8551.00375}$

Villegas, Francisco. y Valderrama, Claudia. (2018). Lógica de gobierno y de gestión en una universidad estatal de Chile. Opción, 34(86), 286-325. Recuperado de https://produccioncientificaluz.org/index.php/opcion/article/view/23847/24280

Webber, Karen. (2018). Institutional research and decision support in higher education: consideration for today and for tomorrow. En Building capacity in institutional research and decision support in higher education (pp. 3-18). Cham: Springer.

Webber, Karen. y Calderon, Angel. (2015). Institutional research and planning: its role in higher education decision support and policy development. En Jeroen Huisman, Harry de Boer, David Dill y Manuel Souto-Otero (Eds.), The Palgrave international handbook of higher education policy and governance (pp. 192-208). Nueva York: Palgrave Macmillan.

Zimmermann, Nicole. (2011). Dynamics of drivers of organizational change. Heidelberg: Gabler. 
Revista indizada en

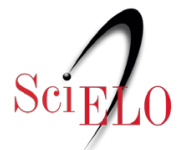

redalyc satindex

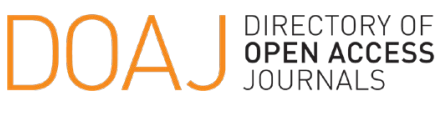

Distribuida en las bases de datos:

- Dialnet

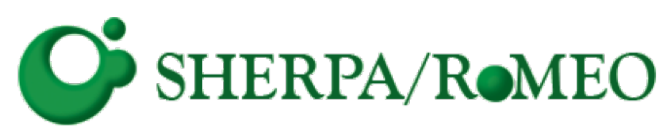

REDIB

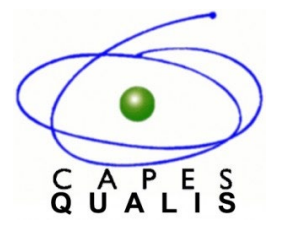

MIAR 